\title{
Sustained release of isoniazid from polylactide microspheres prepared using solid/oil drug loading method for tuberculosis treatment
}

\author{
Limei Zhang ${ }^{\dagger}$, Ying Li ${ }^{\dagger}$, Yun Zhang \& Chunyan Zhu* \\ Institute of Medicinal Plant Development, Chinese Academy of Medical Sciences \& Peking Union Medical College, Beijing 100193, China
}

Received January 26, 2016; accepted March 6, 2016; published online June 7, 2016

\begin{abstract}
Polylactide (PLA) microspheres were prepared using the solid-in-oil (S/O) spray-drying method to achieve the sustained release of a hydrophilic drug for the treatment of tuberculosis, via intratracheal instillation. Isoniazid (IN), a low-molecular-weight hydrophilic drug, was used as a model drug. The effects of various sizes of micronized IN powder, different drug/polymer ratios, spray-drying process parameters, and drug-release characteristics were studied to optimize the manufacturing parameters. A high entrapment efficiency $(87.3 \%)$ was obtained using this method; furthermore, the microspheres were spherical and smooth. They were individually and homogenously distributed, with a mean diameter of $5.6 \mu \mathrm{m}$; furthermore, they showed a satisfactory extended sustained-release phase. After administration of the microspheres to rats, pulmonary drug concentrations were maintained at a relatively stable level for up to 4 weeks.
\end{abstract}

isoniazid, polylactide microspheres, sustained release, solid/oil spraying method, intratracheal intubation

Citation: Zhang, L., Li, Y., Zhang, Y., and Zhu, C. (2016). Sustained release of isoniazid from polylactide microspheres prepared using solid/oil drug loading method for tuberculosis treatment. Sci China Life Sci 59, 724-731. doi: 10.1007/s11427-016-5051-4

\section{INTRODUCTION}

Tuberculosis (TB) is a chronic infectious disease caused by the tubercle bacillus. Despite the development of treatment programs and various anti-TB drugs, TB remains a globally serious infectious disease (Nahid and Menzies, 2012). Since most of the TB manifestations are observed mainly in the respiratory system, pulmonary drug delivery has received increasing attention (Kulik, 1980; Misra et al., 2011; Pandey and Khuller, 2005). The pulmonary delivery of drugs via intratracheal instillation has been increasingly used for the clinical treatment of TB (Fu et al., 2008; Pourshahab et al., 2011; Zahoor et al., 2005). Using the pulmonary topical delivery route, anti-TB drugs are likely to gather in the lungs more rapidly and at higher concentrations without

$\dagger$ Contributed equally to this work

*Corresponding author (email: cyzhu@implad.ac.cn) first-pass metabolism, which could enhance the bioavailability of drugs at their site of action (Furak et al., 2001). In addition, this route reduces systemic adverse effects (Sadovnikov, 1993). However, one of the main problems associated with intratracheal instillation is that it is difficult to maintain a therapeutic drug concentration for the required duration. Frequent dosing is generally associated with worse compliance to the TB treatment; therefore, developing a sustained-release drug delivery system is particularly important in order to improve the therapeutic efficacy. A standard therapy regime involves chemotherapy using rifampicin (RIP) and isoniazid (IN), supplemented with streptomycin or ethambutol for six to nine months (Somner, 1975).

In previous studies, we designed two kinds of drugdelivery systems, poly(lactic-co-glycolic acid) (PLGA) microspheres in a sodium alginate gel for rifampicin (RFP) delivery (Hu et al., 2012) and ethyl cellulose-chitosan com- 
plex microspheres for rifabutin (RBT) delivery (Feng et al., 2013). These delivery systems prolonged drug retention in the lungs, which lead to the maintenance of the therapeutic drug concentration for at least $24 \mathrm{~d}$. Compared to the lipophilic drugs (RFB and RIP), it is more difficult to maintain a sustained delivery of the low-molecular-weight hydrophilic drug, IN, in the lungs.

Polylactide (PLA) is widely used to prepare sustained delivery systems; the most common manufacturing method is the double emulsification $(\mathrm{W} / \mathrm{O} / \mathrm{W}$ ) procedure (Gallarate et al., 2009; Liu et al., 2006; Mun et al., 2010; Yin et al., 2006). Currently, microspheres loaded with hydrophilic drugs, especially those with, low molecular weights, still suffer from two major problems; low drug loading efficiency and burst release behavior (Freiberg et al, 2004; Huang and Brazel, 2001; Park et al, 1992a, b; Wang et al, 1991). The main reasons for the problems were the migration of the drug, porous structure of microspheres, and heterogeneous distribution of the drug in the polymer matrix. Based on these factors, extensive research has been carried out.

In this study, PLA microspheres loaded with IN (PLA-IN-MS) were prepared using the solid-in-oil (S/O) spray-drying method. Unlike in the $\mathrm{W} / \mathrm{O} / \mathrm{W}$ emulsion method, micronized IN powder was used instead of a drug solution. Therefore, there was no outer water phase, which helps to eliminate the osmotic pressures and decreases drug migration; therefore, it may improve the drug loading efficiency. In order to uniformly disperse the drug in the organic solvent, IN powder was micronized via ball milling. The in vitro/in vivo release behaviors were studied to clarify the sustained-release properties of the microspheres.

\section{RESULTS AND DISCUSSION}

Currently, inhalation and intratracheal instillation are two techniques used for the pulmonary administration of drugs (Coowanitwong et al., 2008; Yoshida et al., 2006). However, pharyngeal deposition associated with the inhalation route may lead to difficulties in measuring the dose inside the lungs, and side effects associated with the systemic circulation (Tomoda et al., 2008; Tsapis et al., 2003). In contrast, an intratracheal instillation is a simple, quantifiable way to achieve pulmonary drug delivery, and has been increasingly applied in the clinical treatment of pulmonary tuberculosis.

Microspheres have been widely reported as one of the better formulations for pulmonary administration. However, microspheres prepared using the W/O/W method (Gallarate et al., 2009; Liu et al., 2006; Mun et al., 2010; Yin et al., 2006) have two major problems: a low drug loading efficiency and burst release behavior (Freiberg et al., 2004; Huang and Brazel, 2001; Park et al., 1992a, b; Wang et al., 1991).

In order to restrict drug diffusion, the $\mathrm{S} / \mathrm{O} / \mathrm{W}$ emulsion method was used in this study. This emulsion method has been used in protein and peptide delivery systems. Owing to the low thermal energy state of the solid state, drug particles have a less tendency to migrate from the oil phase to aqueous phase and, therefore, a higher drug loading efficiency was expected.

\section{Preparation of micronized IN powder}

In our previous study, we found that the size of the IN powder particles affected the loading capacity (LC) and entrapment efficiency (EE); smaller powder particles resulted in higher LC and EE values.

Factors including the rotation speed, milling time, different IN/ball weight ratios, and the volume of dichloromethane (DCM) were studied for their possible influences on the particle size of the micronized IN powder, in order to optimize the preparation process, as reported in Table 1. A smaller particle size was observed at a milling speed of 650 rpm for $2 \mathrm{~h}$, with $3 \mathrm{~mL}$ of DCM and an IN/ball weight ratio of $0.5 / 30$. It was shown that the particle size decreased when the grinding time was prolonged from 1 to $2 \mathrm{~h}$; however, the particle size increased when the grinding time was prolonged further, which resulted from inverse crushing. Inverse crushing is a phenomenon where particles agglomerate together, eliciting an apparent size increase when the milling time exceeds a certain period of time. Therefore, the appropriate extension of the milling time can improve the grinding efficiency; however, further extension will not only decrease the milling efficiency, but also contribute to the inverse crushing phenomenon. When the other parameters were kept constant, the particle size reduced with a decreasing IN/ball ratio. With the ratio of ball increasing, the probability of the ball affecting the IN particles increased; furthermore, the energy transferred to the IN particles increased, resulting in smaller-sized particles. Adding DCM significantly decreased the particle size of the IN powder. We found that the IN powder mostly sank to the bottom of the tank without the addition of DCM; moreover, the balls were on top of the powder, which meant that the impaction of the balls on the powder was insufficient. After the addition of DCM, the IN powder adhered to the balls during the milling process and sufficient impaction was achieved. Another reason for the decreased IN powder particle size is that the crushing process can generate new surfaces upon which the surrounding medium can adsorb. This would reduce the particle strength and increase their deformation, resulting in the further breakdown of the particles. Conversely, more work is required to produce new surfaces in a vacuum than in liquid medium. The optimized processing parameters for the IN powder were as follows: $0.5 \mathrm{~g}$ of IN and a $2 \mathrm{~h}$ milling time, $30 \mathrm{~g}$ balls, and $3 \mathrm{~mL}$ of DCM.

\section{Preparation of microspheres}

Previous research (Nahid and Menzies, 2012) indicated that when the microspheres were prepared using the $\mathrm{W} / \mathrm{O} / \mathrm{W}$ method, hydrophilic drugs exhibited a significant tendency 
Table 1 Effects of rotation speed, milling time, amount of isoniazid (IN) and balls, and the volume of DCM on the particle size of micronized IN powder

\begin{tabular}{cccccc}
\hline Rotation speed $(\mathrm{rpm})$ & Milling time $(\mathrm{h})$ & $\mathrm{IN}(\mathrm{g})$ & Balls $(\mathrm{g})$ & DCM $(\mathrm{mL})$ & Particle size $(\mu \mathrm{m})$ \\
\hline 450 & 2 & 1 & 30 & 3 & $4.560 \pm 0.583$ \\
550 & 2 & 1 & 30 & 3 & $1.560 \pm 0.352$ \\
650 & 2 & 1 & 30 & 3 & $0.683 \pm 0.267$ \\
650 & 3 & 1 & 30 & 3 & $1.206 \pm 0.372$ \\
650 & 1 & 1 & 30 & 3 & $1.887 \pm 0.342$ \\
650 & 2 & 0.5 & 30 & 3 & $0.161 \pm 0.102$ \\
650 & 2 & 1 & 20 & 3 & $0.445 \pm 0.136$ \\
650 & 2 & 1 & 10 & 2 & $2.262 \pm 0.353$ \\
650 & 2 & 1 & 30 & - & $0.534 \pm 0.336$ \\
650 & 2 & 1 & 30 & $4.762 \pm 0.103$ \\
\hline
\end{tabular}

Table 2 Effect of the isoniazid particle size and drug/polylactide (PLA) mass ratio on the entrapment efficiency (EE) and loading capacity (LC) of PLA-IN-microspheres (mean $\pm \mathrm{SD}, n=3$ )

\begin{tabular}{|c|c|c|c|c|}
\hline No. & IN/PLA & Size of IN (nm) & $\mathrm{LC} \%$ & $\mathrm{EE} \%$ \\
\hline 1 & $40 / 800$ & $1560 \pm 0.352$ & $3.86 \pm 0.26$ & $76.81 \pm 2.06$ \\
\hline 2 & $40 / 800$ & $683 \pm 0.267$ & $4.61 \pm 0.26$ & $80.46 \pm 1.85$ \\
\hline 3 & $40 / 800$ & $161 \pm 0.106$ & $5.43 \pm 0.26$ & $89.32 \pm 1.21$ \\
\hline 4 & $60 / 800$ & $161 \pm 0.106$ & $7.23 \pm 1.08$ & $84.30 \pm 2.38$ \\
\hline 5 & $80 / 800$ & $161 \pm 0.106$ & $9.16 \pm 0.96$ & $80.17 \pm 1.59$ \\
\hline
\end{tabular}

to migrate from the inner water phase to the outer water phase during the preparation process, resulting in a low drug loading efficiency. Moreover, the migration of water that carries a drug during drying and storage may lead to a heterogeneous drug distribution in the polymer matrix, which increases the probability of a burst release.

Dutt and Khuller controlled microsphere pores by adjusting the concentrations of PLGA and poly(vinyl alcohol) (PVA) (Dutt and Khuller, 2011); they found that the sustained release of IN was prolonged with the incorporation of sphere porosity. Although the hardened PLGA microspheres met the sustained-release standard, the percentage entrapment of IN was only $10 \%-11 \%$. Ito et al. prepared IN-PLGA microspheres by freezing the inner water phase of the primary $\mathrm{W} /\left(\mathrm{O}_{1}+\mathrm{O}_{2}\right)$ emulsion at $-10^{\circ} \mathrm{C}$, followed by the evaporation of the solvent from the secondary $\mathrm{W} /\left(\mathrm{O}_{1}+\mathrm{O}_{2}\right) / \mathrm{O}_{3}$ emulsion (Ito et al., 2011). This procedure eliminated the rapid diffusion of the drug to the outer phase. However, although the drug loading efficiency was improved to approximately $20 \%$, the preparation process is complicated and difficult to control. Thus, in order to improve the loading efficiency and reduce the burst release, it is necessary to restrict the migration of the drug.

In this study, PLA microspheres loaded with IN (PLA-IN-MS) were prepared using the solid-in-oil (S/O) spray-drying method and an EE above $80 \%$ was achieved (Table 2). Unlike in the $\mathrm{W} / \mathrm{O} / \mathrm{W}$ emulsion method, micronized IN powder was used instead of a drug solution and there was no outer water phase, which could be helpful for eliminating the osmotic pressures and decreasing drug migration.

The traditional spray-dryer that is reported in most of the literature uses pressure nozzles and rotary atomizers or a two-fluid nozzle for forming spray droplets. The droplets in the gas flow through an L drying chamber, and are collected in a cyclone collector. During the process, collisions between droplets and the tube wall easily occur due to the strong flow of gas. It is difficult to collect microspheres from the cyclone collector, and the particles are irregular in shape. A Nano Spray Dryer B-90 (BÜCHI Labortechnik AG, Switzerland) was used in this study, which utilizes a vibrating mesh technology for the generation of fine droplets. The spray-drying process is delicate and the size of droplets is mainly controlled by the size of the hole. Furthermore, the spray-dryer has a vertical configuration, facilitating direct and straight-down collection of the particles into the collector, which helps to minimize the collision between the microspheres and the side walls, and results in a mostly spherical particle shape.

The results showed that a smaller drug particle size contributed to a higher EE and drug loading. Therefore, IN powder with a particle size of approximately $0.16 \mu \mathrm{m}$ was used in the next studies.

As shown in Table 2, the LC rose as the proportion of IN to PLA increased; however, the EE dropped. We found that with an increase in the IN particle ratio, the residue of drug particles left on the bottom of the bottle increased. It was thought that the ability of the DCM solution containing PLA to support IN powder was limited. In addition, with the increase of the IN particle ratio, aggregation occurred between the powder particles due to electrostatic adsorption. These aggregates were then deposited on the bottom of the bottle, causing the EE to reduce.

The optimized parameters for the PLA-IN-MS were as follows: an IN/PLA ratio of 60/80 and an IN powder particle size of approximately $161 \mathrm{~nm}$. 


\section{Morphology and particle size distribution of micro- spheres}

Most polymeric microspheres prepared via the spray-drying technique have been reported to have an irregular spherical morphology with pores on the surface (Bodmeier and Chen, 1988; Gavini et al., 2004). However, microspheres with spherical and smooth surfaces were obtained in our study, as shown in Figure 1. This morphology was mainly attributed to the distinctive spray and collection mechanism of the Nano Spray Dryer B-90 that owns three patented technologies (a Piezo electric spray head, a patented heating system, and an electrostatic particle collector), which enabled the production of small particles and reduced the R\&D cost owing to small sample volumes and higher yields.

Furthermore, an enlarged view of one microsphere (Figure $1 \mathrm{~B})$ revealed that there were no obvious pores and cracks on the surface of the microsphere. The mean particle size of the microspheres was approximately $5.6 \mu \mathrm{m}$.

\section{Differential scanning calorimetry (DSC) measurements}

DSC curves of microspheres loaded with IN were drawn in comparison with PLA microspheres (Blank-MS), IN, and their physical mixture (PLA-IN-MIX; Figure 2).

Ball milling is a high-speed operation and the drug crystal form may change during the process; furthermore, degradation and oxidation may occur owing to the heat production as a result of high-speed grinding (Cai, 2004). For these reasons, we investigated the effect of the grinding process on the stability of the drug. The DSC endotherm of the IN sample shows a sharp melting endotherm at $175^{\circ} \mathrm{C}$, and no significant differences were observed between micronized and non-micronized IN (Figure 2). These results indicate that crystal transformation did not occur during the micronization process; therefore, it can be used as a manufacturing step to reduce the particle size of IN, without changing its physical form.

The blank microspheres have one endothermic peak $\left(55^{\circ} \mathrm{C}\right)$, the physical mixture of the drug and blank microspheres has two endothermal peaks $\left(55^{\circ} \mathrm{C}\right.$ and $\left.175^{\circ} \mathrm{C}\right)$ corresponding to PLA blank microspheres and the drug, respectively. However, the typical endothermic peaks of IN were not observed in the curve of the PLA-IN-MS, which illustrates the formation of microspheres and high EE of IN.

\section{In vitro drug release}

The in vitro release of IN from the loaded microspheres was studied for $512 \mathrm{~h}$ at $\mathrm{pH} 7.4$ in phosphate-buffered saline (PBS). In this study, we compared the release rate of IN from microspheres (PLA-IN-MS) and the physical mixture (PLA-IN-MIX; Figure 3A).

It was demonstrated that the in vitro release rate of IN from microspheres was significantly slower than that from the physical mixture. As shown in Figure 3A, the cumulative release percentages of IN from the PLA-IN-MS and PLA-IN-MIX were compared, with $8.97 \%$ and $76.12 \%$ released in $0.5 \mathrm{~h}, 16.1 \%$ and $95.04 \%$ in $2 \mathrm{~h}$, and $19.41 \%$ and $98.01 \%$ in $4 \mathrm{~h}$, respectively. Almost more than $90 \%$ of the IN was released from the physical mixture within $4 \mathrm{~h}$, while there was a sustained release from the PLA-IN-MS, with only $9 \%$ of the drug released within the first $0.5 \mathrm{~h}$, and a significantly reduced burst release.

The microsphere suspension was vortex-mixed before administration, so the effect of this vortex-mixing was also studied. Compared to PLA-IN-MS, a faster release profile of IN was observed from the PLA-IN-MS after vortex-mixing, with $32 \%$ of the drug released within the first $0.5 \mathrm{~h}$. It is reasonable to infer that the strong shear stress of the vortex-mixing carried surface-adhered drug from the microspheres to the medium and, therefore, enhanced drug dissolution. Furthermore, the shear stress also separated the microspheres, with a tremendously increased specific contact area between the microspheres and dissolution medium, which resulted in the higher drug dissolution rate.

As shown in Figure 3B, the percentage of IN released was $34.63 \%$ after $24 \mathrm{~h}$, followed by a gradual and sustained release up to $70.12 \%$ after $552 \mathrm{~h}$. The drug release behavior showed a biphasic pattern indicated by its initial quick release, followed by a very slow release. This initial quick release could be attributed to the vortex-mixing process of the microsphere suspension, which may facilitate drug diffusion from the microsphere surface layer to the medium. After this initial period, the release may have occurred due to the diffusion of the drug from the PLA matrix of the microspheres into the dissolution medium.

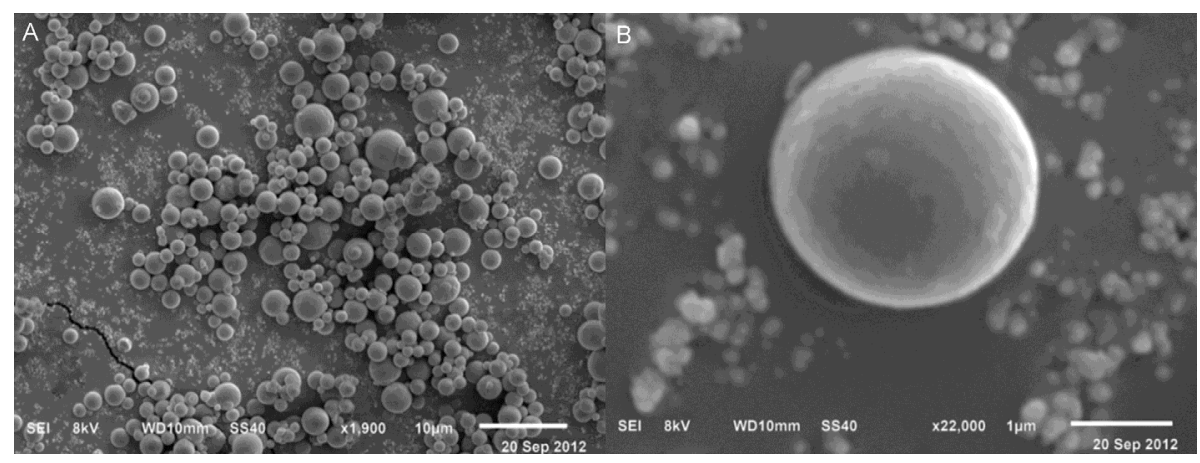

Figure 1 Scanning electron microscopy images of polylactide-isoniazid microspheres. 


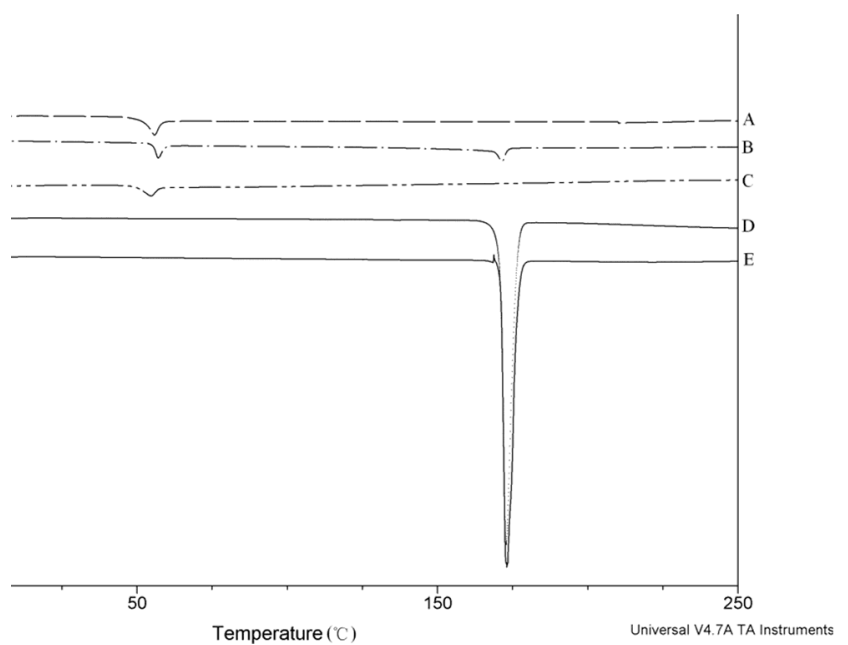

Figure 2 Differential scanning calorimetry study. A, Blank polylactide (PLA) microspheres (MS); B, PLA-IN-MIX; C, PLA-IN-MS; D, micronized IN; E, IN
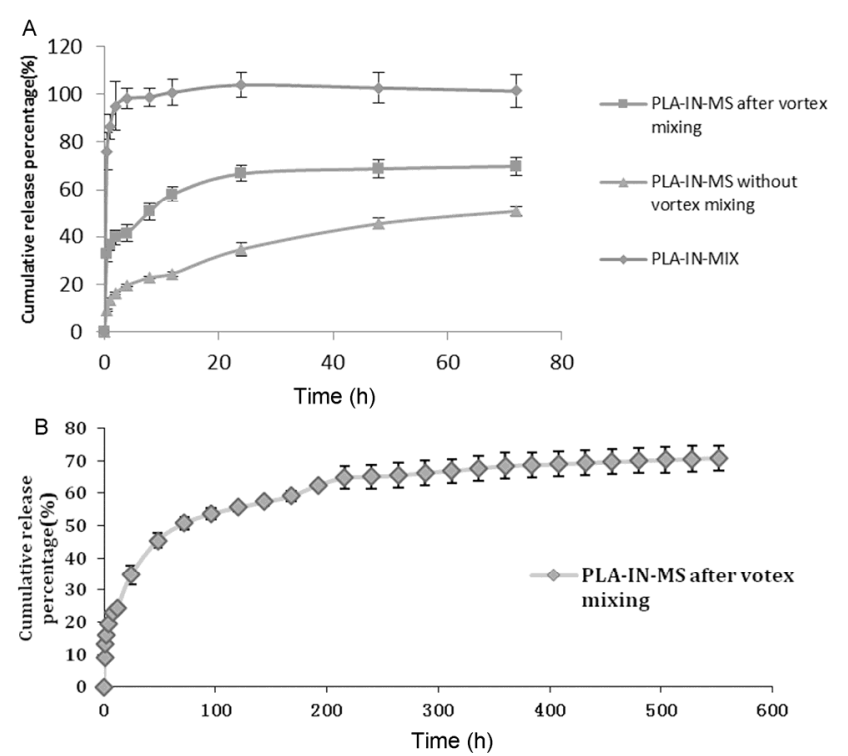

Figure 3 In vitro release study ( $n=6$ ). A, In vitro drug release of isoniazid (IN)-loaded microspheres (MS) after vortex-mixing, IN-loaded MS without vortex-mixing and a polylactide (PLA)-IN-physical mixture (MIX); B, In vitro drug release of IN-loaded MS after vortex-mixing for $512 \mathrm{~h}$.

\section{In vivo drug release}

The bronchoalveolar lavage (BAL) method was used to extract free IN from the lungs of treated rats, as described in the MATERIALS AND METHODS. After the administration of the PLA-IN-MS, the lungs were separated and injected with PBS. The lavage fluid was collected and the free drug was extracted using DCM.

As shown in Figure 4A, the concentration of IN immediately reached $2,659.7 \mathrm{ng} \mathrm{mL}^{-1} 5$ min after administration of the physical mixture of PLA and IN (PLA-IN-MIX), which was almost ten times more than the maximum drug concentration of the test group. The drug concentration then sharply decreased to $23.9 \mathrm{ng} \mathrm{mL^{-1 }}$ after $30 \mathrm{~min}$. After $2 \mathrm{~h}$, no more drug was detected in the physical mixture samples, whereas IN was still releasing slowly from the PLA microspheres.

$\mathrm{A} \mathrm{C}_{\max }$ of $244.8 \mathrm{ng} \mathrm{mL}^{-1}$ was immediately achieved $0.5 \mathrm{~h}$ after administration of the PLA-IN-MS (Figure 4A). The concentration decreased to $80.0 \mathrm{ng} \mathrm{mL}^{-1}$ after $24 \mathrm{~h}$, followed by a plateau level of approximately $60 \mathrm{ng} \mathrm{mL}^{-1}$ that lasted over $14 \mathrm{~d}$ (Figure 4B), which then gradually decreased to $32.8 \mathrm{ng} \mathrm{mL}^{-1}$ after $28 \mathrm{~d}$. Both of these concentrations were higher than the minimal inhibitory concentra-

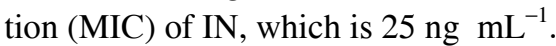

The in vitro release study revealed that vortex-mixing affected the drug release; therefore, the intense vortex-mixing used for the preparation of the PLA-IN-MS suspension is probably responsible for the initial release peak demonstrated in the in vivo experiment. Although an initial burst release was observed, the MS exhibited a sustained release for up to 4 weeks, which is promising for reducing the frequency of drug administration and improving the therapeutic efficacy of the intratracheal treatment of TB.

\section{MATERIALS AND METHODS}

\section{Materials}

IN was purchased from Sigma-Aldrich, USA. PLA with a viscosity of $0.49 \mathrm{dL} \mathrm{g}^{-1}$ (in $\mathrm{CHCl}_{3}$ at $25^{\circ} \mathrm{C}$ ) was obtained
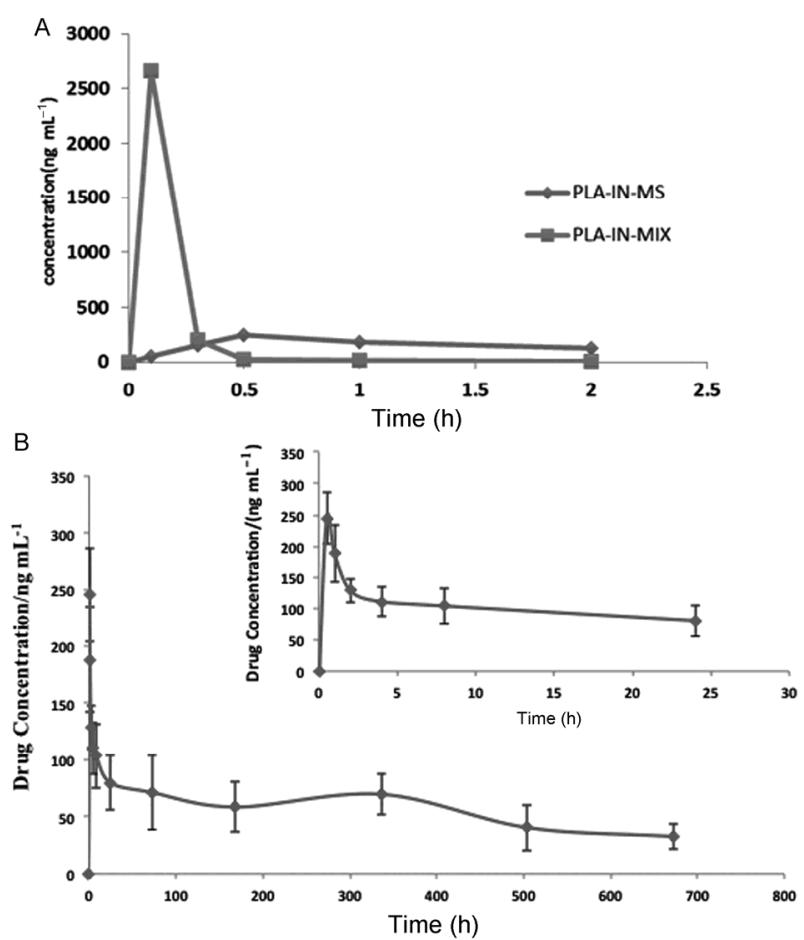

Figure 4 Pulmonary isoniazid (IN) concentration vs. time profiles of IN-polylactide (PLA) microspheres (MS) following intratracheal instillation to rats $($ mean $\pm \mathrm{SD}, n=6)$. 
from the Shandong Institute of Medical Instruments, China. IN (99.5\% purity) standard and internal standard (I.S.) gatifloxacin $(98.0 \%$ purity) were purchased from the National Institute for Food and Drug Control, China. DCM was purchased from the Beijing Chemical Plant, China. Methanol was of HPLC grade (Dikma, USA). Formic acid (AR grade) was purchased from Yongda Chemical Reagent Factory of Tianjin, China.

\section{Animals}

Male Sprague-Dawley (SD) rats weighing from 250 to 300 $\mathrm{g}$ were obtained from Vital River Laboratories, China. Before the study, the rats were allowed to acclimatize to the environment for $7 \mathrm{~d}$. All studies involving animals were performed in accordance with the Guide for the Care and Use of Laboratory Animals.

\section{Preparation of micronized IN powder}

Micronized IN powder was prepared using the ball-milling method with a planetary ball miller (RETSCH PM100, Germany). Alumina balls (2.7 mm diameter), IN, and DCM were loaded into a $25 \mathrm{~mL}$ stainless steel jar, and then ground at low (450 rpm), medium (550 rpm), and high (650 rpm) speeds. The effect of different amounts of IN, balls, and DCM were also studied. The micronized IN powder was collected after the DCM was evaporated. The particle size of the micronized IN powder was analyzed using a Malvern Zetasizer Nano ZS90 (Malvern Instruments, UK).

\section{Preparation of microspheres}

Drug-loaded microspheres (PLA-IN-MS) were prepared using the spray-drying method. Micronized IN powder was dispersed in DCM under sonication for $5 \mathrm{~min}$; subsequently, PLA was added and sonicated until the PLA dissolved. The suspension was then spray-dried using a Nano Spray Dryer B-90 (BÜCHI Labortechnik AG, Switzerland). The operating conditions were kept constant as follows: $T_{\mathrm{m}}=100^{\circ} \mathrm{C}$, feed rate $=15 \mathrm{~mL} \mathrm{~h}^{-1}$, and drying gas flow rate $=100 \mathrm{~L} \mathrm{~min}^{-1}$. Under these conditions, the microspheres were sufficiently dried and less microspheres remained on the wall of the structure. The spray mesh used in this study was a membrane with $7.0 \mu \mathrm{m}$-sized holes.

Blank-MS were prepared using the same method as described for the PLA-IN-MS, without the addition of IN.

A physical mixture of IN and Blank-MS, PLA-IN-MIX, was prepared by mixing IN micronized powder and Blank-MS at the same ratio of IN to PLA as that used for the PLA-IN-MS.

\section{Particle size analysis}

Most of the sizes of the particles that made up the micronized IN powder were at the nanometer level, thus, a Malvern Zetasizer Nano ZS90 (Malvern Instruments, UK) was used, which has a measuring range from $0.3 \mathrm{~nm}$ to 10 $\mu \mathrm{m}$. The specific operation of the instrument was as fol- lows: $10 \mathrm{mg}$ of microspheres and an equal amount of sodium lauryl sulfate were mixed, and then this mixture was ultrasonically dispersed in $4 \mathrm{~mL}$ of distilled water. Finally, the suspension was put into the sampling beaker of the instrument and analyzed. IN powder was ultrasonically dispersed in DCM, and then this suspension was analyzed.

\section{Scanning electron microscopy (SEM)}

The microscopic shape and surface morphology of the microspheres were observed using SEM (JSM-6510, Japan). Microspheres were dispersed on aluminum stubs and then sputter-coated with platinum particles for $120 \mathrm{~s}$. The samples were then observed at an accelerating voltage of $15 \mathrm{kV}$, with the observation magnitude set at $10 \mathrm{~mm}$.

\section{DSC}

Microspheres loaded with IN were characterized using DSC (Q200, TA Instruments, USA). Five samples were prepared in this study: IN, micronized IN, Blank-MS; PLA-IN-MS, and PLA-IN-MIX. Five milligrams of each sample was heated up from $0^{\circ} \mathrm{C}$ to $250^{\circ} \mathrm{C}$ at a speed of $10^{\circ} \mathrm{C} \mathrm{min}^{-1}$. An empty sample pan was used as a reference.

\section{High-performance liquid chromatography (HPLC)}

A Shimadzu Class VP HPLC system (Japan) with a Thermo C18 column $(10 \mathrm{~mm} \times 4.6 \mathrm{~mm} ; 2.4 \mu \mathrm{m})$ combined with a UV detector was used for the drug content analysis. Analytical methods were developed according to the National Pharmacopoeia II. IN was detected at a wavelength of $278 \mathrm{~nm}$. The mobile phase comprised a mixture of methanol and $0.02 \mathrm{~mol} \mathrm{~L}^{-1} \mathrm{Na}_{2} \mathrm{HPO}_{4}$ solution $(\mathrm{pH} 6.45)$ at a ratio of $4: 96$ $(\mathrm{v} / \mathrm{v})$. The flow rate was set at $0.5 \mathrm{~mL} \mathrm{~min}^{-1}$, the column temperature was $40^{\circ} \mathrm{C}$, and the injection volume was $20 \mu \mathrm{L}$.

\section{High performance liquid chromatography-tandem mass spectrometry (HPLC-MS/MS)}

The HPLC-MS/MS system consisted of a 3200 Q TRAP® mass spectrometer (Applied Biosystems Inc., USA) equipped with an electrospray ionization (ESI) source system and an Agilent 1200 HPLC system (Agilent Technologies, USA). Chromatographic separation of the analytes was performed on a Thermo $\mathrm{C}_{18}$ column $(10 \mathrm{~mm} \times 4.6 \mathrm{~mm} ; 2.4$ $\mu \mathrm{m})$ equipped with a Thermo guard column. The mobile phase was a mixture of $0.2 \%$ formic acid in water and methanol $(40: 60 ; \mathrm{v} / \mathrm{v})$, which was used at a flow rate of 0.4 $\mathrm{mL} \min ^{-1}$. The column temperature was kept at $25^{\circ} \mathrm{C}$ throughout the elution process; the sample injection volume was $10 \mu \mathrm{L}$. The ESI source was operated in the positive mode and the final optimized conditions were as follows: nebulizer gas (Gas 1) 60 psi, auxiliary gas (Gas 2) 40 psi, spray voltage $5.5 \mathrm{kV}$, and turbo ion spray temperature $400^{\circ} \mathrm{C}$; cone voltage, $31 \mathrm{~V}$ for IN and $41 \mathrm{~V}$ for I.S.; and collision energy, $17 \mathrm{eV}$ for IN and $43 \mathrm{eV}$ for I.S. The quantification analysis was performed under the multiple reac- 
tion monitoring (MRM) mode using the following transitions: IN $\mathrm{m} / \mathrm{z}$ 138.2-121.2 and gatifloxacin (I.S.) $\mathrm{m} / \mathrm{z}$ 376.2-261.0. All data were processed using the MassChrom software (version 1.4, Sciex, USA).

\section{Determination of the IN EE and LC}

The EE and LC were measured by extracting the IN from the microspheres. IN was extracted from the microspheres with water containing $25 \%$ DCM using ultrasonication for 2 min. The measurements were repeated in triplicates. The extracted drug was analyzed using HPLC. The drug EE and LC were calculated from the following equations:

Loading capacity $(\%)=($ weight of drug in microspheres/weight of microspheres $) \times 100 \%$

Encapsulation efficiency $(\%)=($ actual drug encapsulated/theoretical drug encapsulated) $\times 100 \%$.

\section{In vitro drug release}

The in vitro release test was carried out at $\mathrm{pH} 7.4$ in PBS solution at $37 \pm 0.5^{\circ} \mathrm{C}$. Twenty milligrams of PLA-IN-MS was suspended in the dialysis tubes with $3 \mathrm{~mL}$ of release medium. They were then transferred to an Erlenmeyer flask containing $200 \mathrm{~mL}$ of medium, incubated in a water bath (SHA-B (A), Jintankexi apparatus Co. Ltd., Jiangsu), and shaken at 100 strokes $\min ^{-1}$ for $8 \mathrm{~d}$. At pre-determined time intervals, $0.5 \mathrm{~mL}$ aliquots of the solutions were withdrawn and filtered through $0.45 \mu \mathrm{m}$ filters. The sample volumes were replaced with equal volumes of the fresh medium. The IN released from the PLA-IN-MS was quantified using HPLC. Three tests were taken for each sample and the mean values were used as the final results.

\section{In vivo release}

SD rats were divided into two groups. After anesthesia using pentobarbital sodium $\left(60 \mathrm{mg} \mathrm{kg}^{-1}\right)$, the experimental group was administered $0.2 \mathrm{~mL}$ of the PLA-IN-MS suspension via an intratracheal intubation operation. The control group was administered with the same volume of a suspension of the PLA-IN-MIX. The rats were sacrificed at predetermined time intervals, and their tracheae and lung tissues were carefully separated to extract IN according to the BAL method previously reported. A specific amount of PBS was injected into the tracheae and lung tissues, and then withdrawn immediately. The whole process was repeated four times. Methanol $(360 \mu \mathrm{L})$ and $10 \mu \mathrm{L}$ of the internal standard solution $\left(0.96 \mu \mathrm{g} \mathrm{mL}^{-1}\right.$ gatifloxacin) were put into the lavage fluid $(130 \mu \mathrm{L})$, vortex-mixed for $2 \mathrm{~min}$, and centrifuged at $13,000 \times g$ for $5 \mathrm{~min}$. The supernatant was then collected and analyzed using high performance liquid chromatography-tandem mass spectrometry.

\section{Statistical analysis}

Each experiment was conducted in triplicate; the statistical analysis was performed using the SPSS software (version 17.0, SPSS Inc., USA).

\section{CONCLUSION}

In this study, PLA-IN-MS were prepared using the spray-drying technique, which was rapid and easy to control. Instead of a drug solution, the micronized IN powder (with an average particle size of approximately $160 \mathrm{~nm}$ ) was directly dispersed into the oil phase and then this suspension was spray-dried. Water was not introduced into the preparation process; therefore, this method overcomes the problems associated with the migration of water and drug during the emulsion and drying processes. Microspheres prepared using this technique were spherical, with a smooth and distorted morphology. A high EE (87.3\%) was obtained using this method. In the in vivo release test, a relatively stable IN concentration was maintained for up to four weeks in rat lungs. This system has the potential to reduce the frequency of administration and improve the therapeutic efficacy of the intratracheal treatment of TB; therefore, we hope that these results will be helpful for the clinical treatment of pulmonary tuberculosis.

Compliance and ethics The author(s) declare that they have no conflict of interest.

Acknowledgements This study was supported by the 12th Five-Year Important National Science \& Technology Specific Projects, and the National Science \& Technology Major Special Project on the Prevention and Cure of Acquired Immune Deficiency Syndrome and Virus Hepatitis (2012ZX10003009-001-002).

Bodmeier, R., and Chen, H. (1988). Preparation of biodegradable poly (+/-) lactide microparticles using a spray-drying technique. J Pharm Pharmacol 40, 754-757.

Cai, Z. (2004). Preparation and quality control study of isoniazid rifampicin and pyrazinamide tablets. Dissertation for Master Degree. (Wuhan Wuhan University), pp. 1-9.

Coowanitwong, I., Arya, V., Kulvanich, P., and Hochhaus, G. (2008). Slow release formulations of inhaled rifampin. AAPS J 10, 342-348.

Dutt, M., and Khuller, G. (2011). Sustained release of isoniazid from a single injectable dose of poly (DL-lactide-co-glycolide) microparticles as a therapeutic approach towards tuberculosis. Int J Antimicrob Agents $17,115-122$.

Feng, H., Zhang, L., and Zhu, C. (2013). Genipin crosslinked ethyl cellulose-chitosan complex microspheres for anti-tuberculosis delivery. Colloids Surf B Biointerfaces 103, 530-537.

Freiberg, S., and Zhu, X. (2004). Polymer microspheres for controlled drug release. Int J Pharm 282, 1-18.

Fu, Y., Chu, N., Yuan, S., Chen, W., Wang, W., Luo, Y., Xiao, H., and Zhu, L. (2008). The effect of interventional therapy in multimodality treatment on multi-drug resistant pulmonary tuberculosis (in Chinese). Zhonghua Jie He He Hu Xi Za Zhi 31, 95-98.

Furak, J., Trojan, I., Szoke, T., Tiszlavicz, L., Morvay, Z., Csada, E., and Balogh, A. (2001). Surgical intervention for pulmonary tuberculosis: analysis of indications and perioperative data relating to diagnostic and therapeutic resections. Eur J Cardiothorac Surg 20, 722-727.

Gallarate, M., Trotta, M., Battaglia, L., and Chirio, D. (2009). Preparation 
of solid lipid nanoparticles from W/O/W emulsions: preliminary studies on insulin encapsulation. J Microencapsul 26, 394-402.

Gavini, E., Chetoni, P., Cossu, M., Alvarez, M.G., Saettone, M.F., and Giunchedi, P. (2004). PLGA microspheres for the ocular delivery of a peptide drug, vancomycin using emulsification/spray-drying as the preparation method: in vitro/in vivo studies. Eur J Pharm Biopharm 57, 207-212.

Hu, C., Feng, H., and Zhu, C. (2012). Preparation and characterization of rifampicin-PLGA microspheres/sodium alginate in situ gel combination delivery system. Colloids Surf B Biointerfaces 95, 162-169.

Huang, X., and Brazel, C.S. (2001). On the importance and mechanisms of burst release in matrix-controlled drug delivery systems. J Control Release $73,121-136$.

Ito, F., Fujimori, H., Kawakami, H., Kanamura, K., and Makino, K. (2011). Technique to encapsulate a low molecular weight hydrophilic drug in biodegradable polymer particles in a liquid-liquid system. Colloids Surfaces A: Physicochem Eng Aspects 384, 368-373.

Kulik, N.M. (1980). Treatment of pulmonary tuberculosis by the inhalation of aerosols of antibacterial preparations and pathogenetic agents. Probl Tuberk 1, 40-42.

Liu, R., Huang, S., Wan, Y., Ma, G., and Su, Z. (2006). Preparation of insulin-loaded PLA/PLGA microcapsules by a novel membrane emulsification method and its release in vitro. Colloids Surf B Biointerfaces 51, 30-38.

Misra, A., Hickey, A.J., Rossi, C., Borchard, G., Terada, H., Makino, K., Fourie, P.B., and Colombo, P. (2011). Inhaled drug therapy for treatment of tuberculosis. Tuberculosis (Edinb) 91, 71-81.

Mun, S., Choi, Y., Rho, S.J., Kang, C.G., Park, C.H., and Kim, Y.R. (2010). Preparation and characterization of water/oil/water emulsions stabilized by polyglycerol polyricinoleate and whey protein isolate. $\mathrm{J}$ Food Sci 75, E116-E125.

Nahid, P., and Menzies, D. (2012). Update in tuberculosis and nontuberculous mycobacterial disease 2011. Am J Respir Crit Care Med 185, 1266-1270.

Pandey, R., and Khuller, G.K. (2005). Antitubercular inhaled therapy: opportunities, progress and challenges. J Antimicrob Chemother 55, $430-435$.

Park, T.G., Cohen, S., and Langer, R. (1992a). Controlled protein release from polyethyleneimine-coated poly(L-lactic acid)/pluronic blend matrices. Pharm Res 9, 37-39.
Park, T.G., Cohen, S., and Langer, R. (1992b). Poly (L-lactic acid)/pluronic blends: characterization of phase separation behavior, degradation, and morphology and use as protein-releasing matrixes. Macromolecules 25, 116-122.

Pourshahab, P.S., Gilani, K., Moazeni, E., Eslahi, H., Fazeli, M.R., and Jamalifar, H. (2011). Preparation and characterization of spray dried inhalable powders containing chitosan nanoparticles for pulmonary delivery of isoniazid. J Microencapsul 28, 605-613.

Sadovnikov, A.A. (1993). Intrapulmonary instillations in destructive tuberculosis. Grud Serdechnososudistaia Khir 3, 40-42.

Somner, A.R. (1975). Short-course chemotherapy in pulmonary tuberculosis, A controlled trial bythe British Thoracic and Tuberculosis Association. Lancet 1, 119-124.

Tomoda, K., Ohkoshi, T., Nakajima, T., and Makino, K. (2008). Preparation and properties of inhalable nanocomposite particles: effects of the size, weight ratio of the primary nanoparticles in nanocomposite particles and temperature at a spray-dryer inlet upon properties of nanocomposite particles. Colloids Surf B Biointerfaces 64, 70-76.

Tsapis, N., Bennett, D., O’Driscoll, K., Shea, K., Lipp, M.M., Fu, K., Clarke, R.W., Deaver, D., Yamins, D., Wright, J., Peloquin, C.A., Weitz, D.A., and Edwards, D.A. (2003). Direct lung delivery of para-aminosalicylic acid by aerosol particles. Tuberculosis (Edinb) 83 , 379-385.

Wang, H., Schmitt, E., Flanagan, D., and Linhardt, R. (1991). Influence of formulation methods on the in vitro controlled release of protein from poly (ester) microspheres. J Control Release 17, 23-31.

Yin, J., Noda, Y., and Yotsuyanagi, T. (2006). Properties of poly(lactic-co-glycolic acid) nanospheres containing protease inhibitors: camostatmesilate and nafamostatmesilate. Int J Pharm 314, 46-55.

Yoshida, A., Matumoto, M., Hshizume, H., Oba, Y., Tomishige, T., Inagawa, H., Kohchi, C., Hino, M., Ito, F., Tomoda, K., Nakajima, T., Makino, K., Terada, H., Hori, H., and Soma, G. (2006). Selective delivery of rifampicin incorporated into poly(DLlactic-co-glycolic) acid microspheres after phagocytotic uptake by alveolar macrophages, and the killing effect against intracellular Mycobacterium bovis Calmette-Guerin. Microbes Infect 8, 2484-2491.

Zahoor, A., Sharma, S., and Khuller, G. (2005). Inhalable alginate nanoparticles as antitubercular drug carriers against experimental tuberculosis. Int J Antimicrob Ag 26, 298-303.

Open Access This article is distributed under the terms of the Creative Commons Attribution License which permits any use, distribution, and reproduction in any medium, provided the original author(s) and source are credited. 